\title{
The Hamiltonicity of swapped (OTIS) networks built of Hamiltonian component networks
}

\author{
Behrooz Parhami * \\ Department of Electrical and Computer Engineering, University of California, Santa Barbara, CA 93106-9560, USA \\ Received 14 November 2004; accepted 9 May 2005 \\ Available online 21 June 2005 \\ Communicated by F. Dehne
}

\begin{abstract}
A two-level swapped (also known as optical transpose interconnect system, or OTIS) network with $n^{2}$ nodes is built of $n$ copies of an $n$-node basis network constituting its clusters. A simple rule for intercluster connectivity (node $j$ in cluster $i$ connected to node $i$ in cluster $j$ for all $i \neq j$ ) leads to regularity, modularity, packageability, fault tolerance, and algorithmic efficiency of the resulting networks. We prove that a swapped network is Hamiltonian if its basis network is Hamiltonian. This general closure property for Hamiltonicity under swap or OTIS composition replaces a number of proofs in the literature for specific basis networks and obviates the need for proving Hamiltonicity for many other basis networks of potential practical interest.
\end{abstract}

(C) 2005 Elsevier B.V. All rights reserved.

Keywords: Hamiltonicity; Hierarchical network; Interconnection architecture; OTIS network; Parallel processing

\section{Introduction}

A number of structural properties of interconnection networks are of interest in parallel and distributed computation because they lead to important fault tolerance and performance attributes or affect the ease of use. Hamiltonicity of a $p$-node graph $G$, defined as a $p$-node cycle being a subgraph of $G$, is one such property that is important to ensure deadlock freedom in

\footnotetext{
* Tel.: +1 805893 3211; fax: +1 8058933262 .

E-mail address: parhami@ece.ucsb.edu (B. Parhami).
}

some routing algorithms and to allow efficient emulation of linear-array and ring algorithms, among other advantages. Studying general composite interconnection schemes, such as swapped networks, is important in that it allows the derivation of results pertaining to a wide array of network architectures. For example, our proof of Hamiltonicity for a swapped network with a Hamiltonian basis network leads to Hamiltonicity results for a wide array of interconnection networks. This is clearly preferable to proving each network to be Hamiltonian separately, as has been the norm in parallel processing research. For example, the recent 


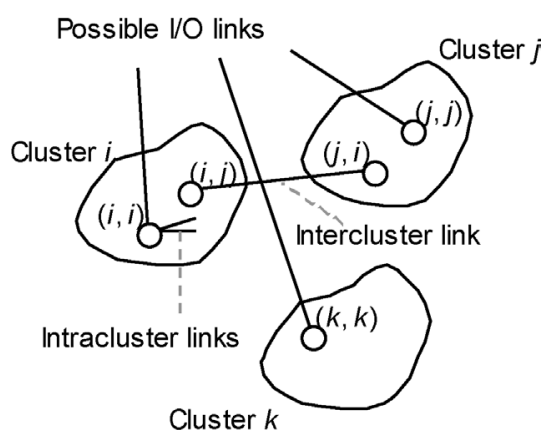

(a)

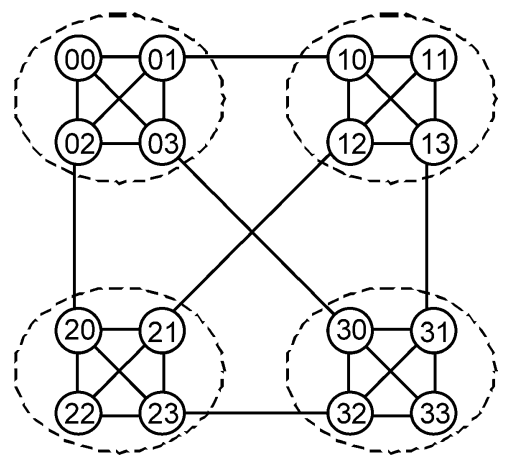

(b)

Fig. 1. The general structure of a swapped network and an example network with the 4-node complete graph as its basis. (a) Intracluster, intercluster, and I/O links. (b) An example 16-node swapped network.

result of $\mathrm{Fu}$ and Chen on the Hamiltonicity of hierarchical cubic networks [1] follows as a special case our result.

Definition 1 (Swapped network, [2]). The swapped network $\operatorname{Sw}(G)$, derived from the $n$-node nucleus or basis graph $G$, is an $n^{2}$-node graph with $n$ copies of $G$ (clusters) numbered 0 to $n-1$, so that node $i$ in cluster $j$ is connected to node $j$ of cluster $i$ for all $i \neq j$ and $0 \leqslant i, j \leqslant n-1$.

\section{Related work}

The author recently discovered, via a tip from an anonymous reviewer, that swapped networks are the same as OTIS (optical transpose interconnection system) architectures, which have been extensively studied by other researchers. Tracing the history of OTIS, the author discovered that its roots go back to 1993, when Marsden et al. published a three-page note in Optics Letters [3] suggesting a topology in which nodes $(i, j)$ and $(j, i)$ are linked via an optical channel. It appears that transfer of the OTIS idea to the computer architecture and parallel processing community occurred, in part, due the $1998 \mathrm{PhD}$ dissertation of C.-F. Wang at University of Florida, under Professor Sartaj Sahni, and publication of its results beginning in the late 1990s [4]. Architectural and some topological considerations for OTIS networks have been studied by Zane et al. [5] and Day and Al-Ayyoub [6], among others.

Concurrent with the developments just cited, and before any reference to OTIS appeared in the com- puter architecture or parallel processing literature, Chi-Hsiang Yeh (a former doctoral student of the author) proposed swapped networks [2] as tools for unifying and extending a number of known hierarchical networks. Prominent among these were the two-level special case of WK-recursive networks [7] (beyond two levels, WK-recursive and swapped networks diverge in structure and do not have much in common), hierarchical cubic networks [8], and recursively fully connected networks [9]. The unification was due to the replacement of complete-graph or hypercube component networks of the prior architectures with an arbitrary graph.

It thus appears that swapped and OTIS architectures have evolved independently and in parallel. Our original paper on swapped networks [2] was followed by hierarchical and recursive versions. Similarly, OTIS networks were studied by many researchers. It is worth noting that "swapped network" is a much more descriptive name than OTIS because many of the algorithms and topological properties discussed in the literature are more or less independent of implementation technology. Reference to optical implementation may in fact have served to discourage researchers not involved in optical computing or optical communications from examining the OTIS results, which may be useful in other contexts.

\section{Hamiltonicity results}

As stated in the introduction, Hamiltonicity is an important and useful property of interconnection net- 


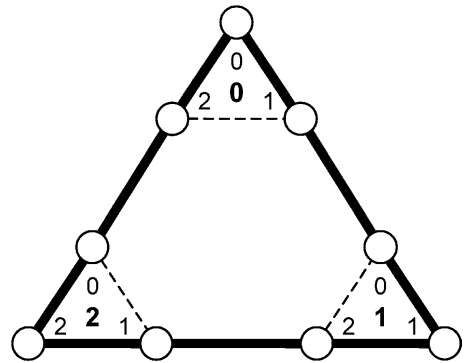

(a)

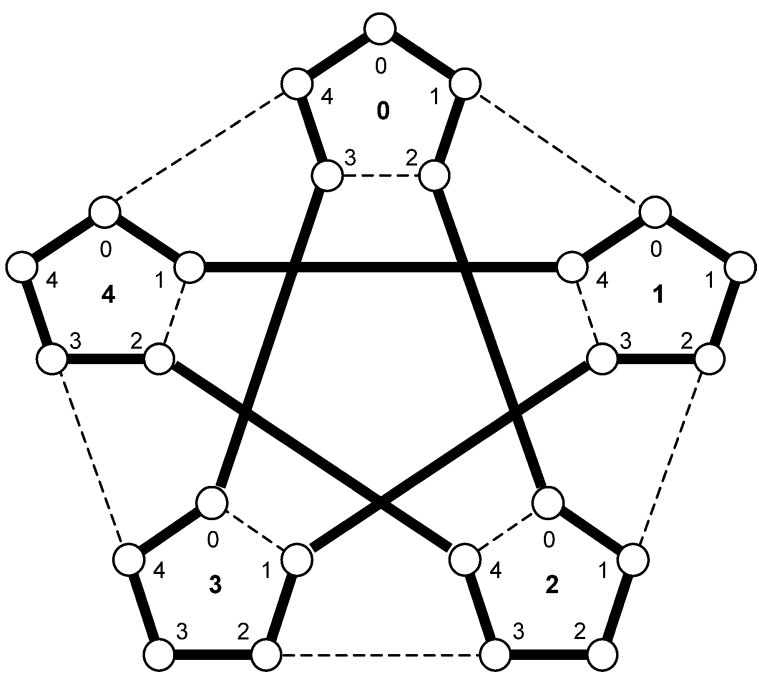

(c)

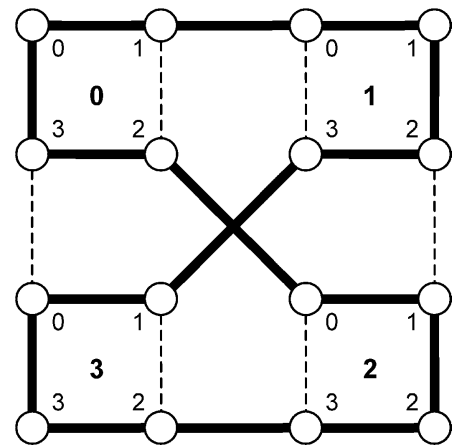

(b)

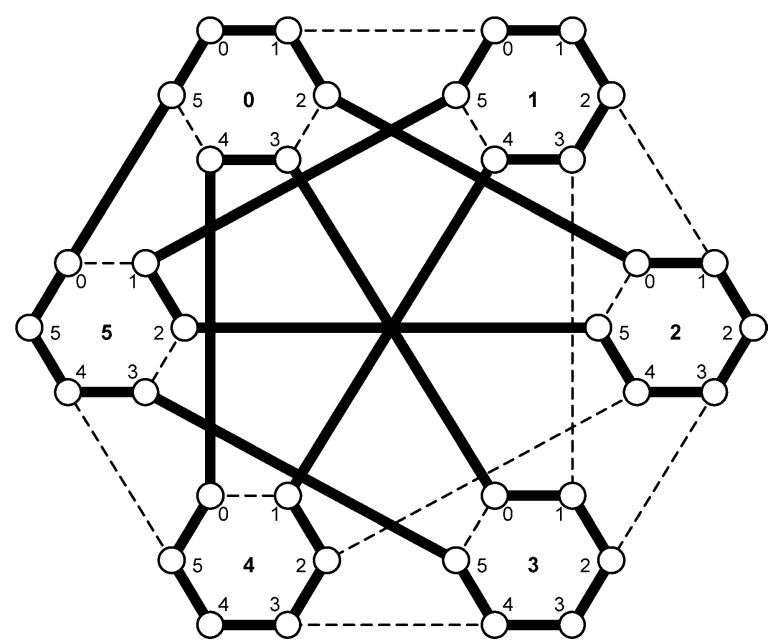

(d)

Fig. 2. Hamiltonian cycle in $\operatorname{Sw}(G)$ whose basis network $G(|G| \leqslant 6)$ is Hamiltonian. Within a cluster, only links belonging to the cluster's Hamiltonian cycle are shown. (a) $n=3$, (b) $n=4$, (c) $n=5$, (d) $n=6$.

works. The related notions of Hamiltonian paths and Hamiltonian connectivity are also of great practical interest. Researchers often go to great lengths to establish the Hamiltonicity and related properties of a particular interconnection network. In this section, we provide a proof that a swapped network $\operatorname{Sw}(G)$ is Hamiltonian if its basis network $G$ is Hamiltonian. This result supersedes a number of results proven in the literature for specific basis networks and obviates the need for proving Hamiltonicity for many other basis networks of potential practical interest.

Definition 2 (Hamiltonian cycle). A Hamiltonian cycle in graph $G$ is a path that leads from a node $u$ back to $u$ and visits each of the other nodes of $G$ exactly once.

Before proving our main theorems, we demonstrate that $\operatorname{Sw}(G)$ is Hamiltonian when $G$ is Hamiltonian and $3 \leqslant n=|G| \leqslant 6$ (see Fig. 2). These cases cover small values of $n(3,4$, and 5), supply the intuition behind the proof for odd $n$, and provide a basis $(n=6)$ for our inductive proof when $n$ is even. Throughout, we will assume that the Hamiltonian cycle visits the nodes in numerical order of their indices: $0,1,2, \ldots, n-1,0$.

Theorem 1 (Hamiltonicity for odd $n$ ). If $G$ contains an odd number of nodes and is Hamiltonian, then so is $\operatorname{Sw}(G)$. 
Table 1

\begin{tabular}{lll}
\hline Cluster & Entry & Exit \\
\hline 0 & $h$ & $h+1$ \\
$h+1$ & 0 & 1 \\
1 & $h+1$ & $h+2$ \\
$h+2$ & 1 & 2 \\
2 & $h+2$ & $h+3$ \\
$h+3$ & 2 & 3 \\
$\vdots$ & & \\
$h-1$ & $2 h-1$ & $2 h$ \\
$2 h$ & $h-1$ & $h$ \\
$h$ & $2 h$ & 0 (back to cluster 0) \\
\hline
\end{tabular}

Proof. Let $n=2 h+1$ and assume that a Hamiltonian cycle goes through nodes $0,1,2, \ldots, 2 h, 0$ of $G$, in order, renumbering the nodes and clusters, if necessary, to accomplish this. Then, Table 1 shows a Hamiltonian cycle that begins and ends in node $h$ of cluster 0 . Each line contains a cluster number, the first node visited in that cluster (entry), and the last node visited (exit). The direction is always backward (toward smaller indices), so that the entry point $h$ and the exit point $h+1$ represent the path $h, h-1, h-2, \ldots, h+2, h+1$ within the cluster.

Note that the first (last) node visited in each cluster has the same index as the (preceding) following cluster, ensuring the availability of intercluster links (see Fig. 2(c)).

Theorem 2 (Hamiltonicity for even $n$ ). If $G$ contains an even number $n=2 h$ of nodes and is Hamiltonian, then so is $\operatorname{Sw}(G)$.

Proof. We prove this by induction on $n$, assuming that an $n$-node cluster's Hamiltonian cycle is $0,1,2$, $\ldots, n-1,0$. The result holds for $n=4$, as easily seen from Fig. 2(b). Our induction basis is $n=6$, for which a Hamiltonian cycle is shown in Fig. 2(d). As part of the basis of our induction, we note that the Hamiltonian cycle of Fig. 2(d) is such that clusters 2 and 3 are the only clusters in which the link between nodes 0 and $n-1=5$ is unused; this property will be maintained throughout. Now assuming that the theorem holds for $n$-node clusters, we construct a Hamiltonian path for the case of $(n+2)$-node clusters, while maintaining the property that the link between nodes 0 and $n+1$ is not part of the Hamiltonian path only in clusters 2 and 3. Start by drawing a Hamiltonian

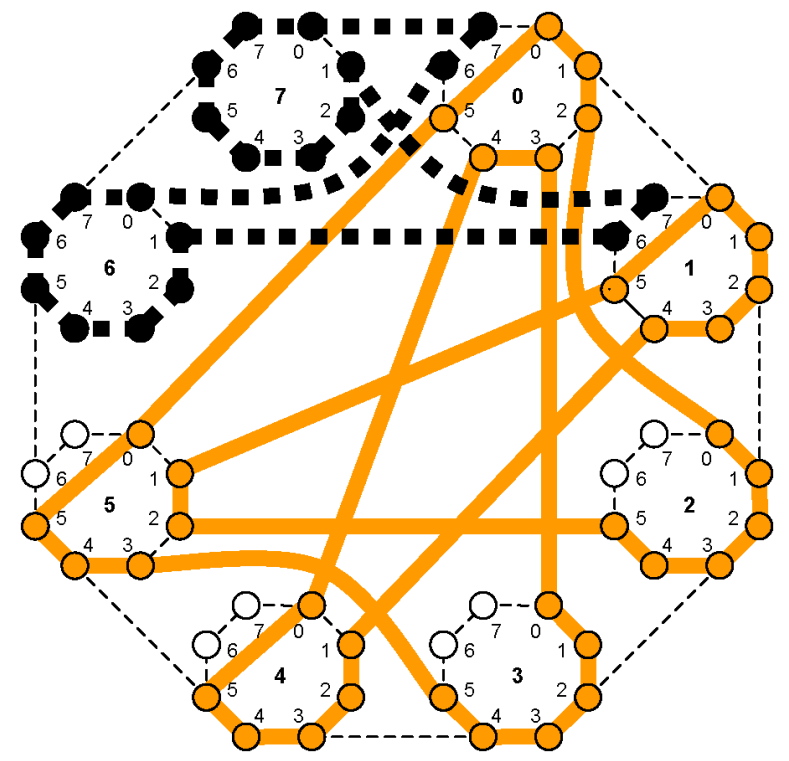

(a)



(b)

Fig. 3. Building a Hamiltonian cycle for $\operatorname{Sw}(G)$, with $|G|=n+2$ (even). (a) Hamiltonian cycle in $S w(G)$, for $|G|=n$. (b) Merging the two cycles of part (a).

path through all the nodes 0 to $n-1$ of clusters 0 to $n-1$, assuming existence of a link between nodes 0 and $n-1$, as would be the case in an $n$-node Hamiltonian cluster (the heavy gray line in Fig. 3(a)). Then draw a cycle encompassing all nodes in clusters $n$ and $n+1$ and nodes $n$ and $n+1$ in clusters 0 and 1 (the 
heavy dotted line in Fig. 3(a)). Next, merge the two cycles of Fig. 3(a) into one via simple modifications in clusters 0 and 1 , using nodes $n$ and $n+1$ in clusters 2 and 3, and modifying the paths within clusters 4 through $n-1$ to cover nodes $n$ and $n+1$ in those clusters (Fig. 3(b)). Note that in the final drawing, the link between nodes 0 and $n+1$ remains unused only in clusters 2 and 3, as postulated in our inductive argument.

\section{Conclusion}

We showed that the Hamiltonicity property is preserved in hierarchical structures built as swapped networks. Our results supersede a number of Hamiltonicity proofs for networks that are special cases of swapped networks and will obviate the need for additional proofs for other basis networks of practical interest. One example is the recent result of $\mathrm{Fu}$ and Chen [1] on the Hamiltonicity of hierarchical cubic networks (swapped networks built from hypercubes) which can be stated as a corollary to our Theorems 1 and 2. Work is in progress on detailed evaluation of swapped networks as interconnection structures for cost-effective, modular, and robust parallel processors [10] and on establishing a number of other general results for such networks.

\section{References}

[1] J.S. Fu, G.H. Chen, Hamiltonicity of the hierarchical cubic network, Theory Comput. Systems 35 (2002) 59-79.

[2] C.-H. Yeh, B. Parhami, Swapped networks: unifying the architectures and algorithms of a wide class of hierarchical parallel processors, in: Proc. Internat. Conf. Parallel and Distributed Systems, 1996, pp. 230-237.

[3] G. Marsden, P. Marchand, P. Harvey, S. Esener, Optical transpose interconnection system architectures, Optics Lett. 18 (1993) 1083-1085.

[4] C.-F. Wang, S. Sahni, Basic operations on the OTIS-mesh optoelectronic computer, IEEE Trans. Parallel Distrib. Systems 9 (1998) 1226-1236.

[5] F. Zane, P. Marchand, R. Paturi, S. Esener, Scalable network architectures using the optical transpose interconnection system, J. Parallel Distrib. Comput. 60 (2000) 521-538.

[6] K. Day, A. Al-Ayyoub, Topological properties of OTIS-networks, IEEE Trans. Parallel Distrib. Systems 13 (2002) 359366.

[7] G. Della Vecchia, C. Sanges, Recursively scalable networks for message passing architectures, in: Proc. Conf. Parallel Processing and Applications, 1987, pp. 33-40.

[8] K. Ghose, K.R. Desai, Hierarchical cubic networks, IEEE Trans. Parallel Distrib. Systems 6 (1995) 427-435.

[9] C.-H. Yeh, B. Parhami, Recursively fully-connected networks: a class of high-performance low-degree interconnection networks, in: Proc. 11th Internat. Conf. Computers and their Applications, 1996, pp. 227-230.

[10] B. Parhami, D.-M. Kwai, Challenges in interconnection network design in the era of multiprocessor and massively parallel microchips, in: Proc. Internat. Conf. Communications in Computing, 2000, pp. 241-246. 